\title{
ILCEA
}

Revue de l'Institut des langues et cultures

d'Europe, Amérique, Afrique, Asie et Australie

$26 \mid 2016$

Mémoire, vérité et justice en Uruguay

\section{La question de la justice et de la vérité en Uruguay devant les Nations unies}

Intervention de Monsieur le Professeur Olivier de Frouville à l'occasion du colloque international « Mémoire, vérité et justice ", les 9 et 10 octobre 2014, organisé par l'ILCEA4

La cuestión de la justicia y de la verdad en Uruguay ante las Naciones Unidas

United Nations and the Issue of Truth and Justice in Uruguay

\section{Olivier de Frouville}

\section{CpenEdition}

\section{Journals}

Édition électronique

URL : http://journals.openedition.org/ilcea/3895

DOI : 10.4000/ilcea.3895

ISSN : 2101-0609

Éditeur

UGA Éditions/Université Grenoble Alpes

Édition imprimée

ISBN : 978-2-84310-334-6

ISSN : 1639-6073

Référence électronique

Olivier de Frouville, "La question de la justice et de la vérité en Uruguay devant les Nations unies », ILCEA [En ligne], 26 | 2016, mis en ligne le 07 juillet 2016, consulté le 19 avril 2019. URL : http:// journals.openedition.org/ilcea/3895; DOI : 10.4000/ilcea.3895

Ce document a été généré automatiquement le 19 avril 2019

(c) ILCEA 


\title{
La question de la justice et de la vérité en Uruguay devant les Nations unies
}

\author{
Intervention de Monsieur le Professeur Olivier de Frouville à l'occasion \\ du colloque international " Mémoire, vérité et justice ", les 9 et \\ 10 octobre 2014, organisé par l'ILCEA4 \\ La cuestión de la justicia y de la verdad en Uruguay ante las Naciones Unidas \\ United Nations and the Issue of Truth and Justice in Uruguay
}

\section{Olivier de Frouville}

Excellence, Mesdames, Messieurs, chers amis, cher Louis,

2 Je voulais tout d'abord vous demander de me pardonner de ne pas être avec vous aujourd'hui.

3 J'aurais énormément aimé participer à ce colloque magnifique, avec un programme et des intervenants passionnants, mais j'ai dû finalement y renoncer, la période étant extrêmement chargée sur le plan universitaire et sur d'autres fronts. Les organisateurs ont très gentiment proposé que je vous transmette ce texte, comportant quelques points de repères sur le droit international et les procédures internationales, pour ce qui a trait à la question des disparitions forcées en Uruguay et, plus largement, à la question du droit à la justice et du droit à la vérité des victimes de violations graves des droits de l'homme pendant la période de la dictature.

4 Je dirai d'abord quelques mots de la manière dont la situation de l'Uruguay a été envisagée pendant plus de 30 ans au sein du Groupe de travail sur les disparitions forcées. Puis je mentionnerai quelques points de droit qui me paraissent importants pour la situation de l'Uruguay, en prenant pour base les travaux du Groupe de travail, mais aussi du Comité contre les disparitions forcées, puisque vous savez qu'en mars 2009, l'Uruguay a ratifié la Convention des Nations unies pour la protection de toutes les personnes contre les disparitions forcées. 


\section{L'Uruguay devant le Groupe de travail sur les disparitions forcées ou involontaires depuis 1980}

5 Rappelons que le Groupe de travail a été créé en 1980 par la Commission des droits de l'homme des Nations unies, remplacée en 2006 par le Conseil des droits de l'homme des Nations unies. Le Groupe de travail est un organe de cinq experts indépendants. Il a principalement deux mandats.

6 Un mandat humanitaire, qui consiste à servir de «canal de communication » entre les familles de disparus et les États concernés. Concrètement le Groupe de travail reçoit des cas de disparitions forcées de la part des familles ou des ONG, les renvoie à l'État en lui demandant de prendre toutes les mesures en vue de localiser la personne, que celle-ci soit vivante ou morte. Le Groupe de travail conserve le cas dans sa base de donnée jusqu'à ce qu'il soit « élucidé».

7 Le Groupe de travail a également un mandat juridique, en ce qu'il surveille l'application par les États de la Déclaration des Nations unies pour la protection de toutes les personnes contre les disparitions forcées. Dans ce cadre, il reçoit des allégations de violations de la Déclaration qu'il adresse aux États, en leur demandant d'apporter des explications.

8 L'ensemble de son activité fait l'objet de rapports publics qui sont présentés au Conseil des droits de l'homme tous les ans.

9 Le Groupe de travail a été saisi dès sa première année d'existence de la situation en Uruguay. Dans son premier rapport (E/CN.4/1435), le Groupe indique avoir reçu « une trentaine de communications", concernant "des ressortissants uruguayens qui ont disparu après avoir été enlevés à l'étranger, particulièrement en Argentine, par des personnes qui appartiendraient aux forces de sécurité uruguayennes ou par des groupes en partie composées de ces dernières. Douze autres communications concernent des personnes ayant disparu après avoir été arrêtées en Uruguay par les forces de sécurité uruguayennes » (\$152). Le Groupe de travail relève que, selon les communications, ces personnes seraient détenues dans plusieurs centres de détention en Uruguay, dont des centres de détention secret, tels que "El Jardin » et "Automotores Orletti » à Buenos Aires. Le Groupe de travail a transmis une partie des cas au Gouvernement et demande à pouvoir contacter directement les personnes concernées. La Mission permanente à Genève fait savoir au Groupe qu'elle " examine sa demande ", mais qu'il n'est pas possible d'accueillir le Groupe à l'heure actuelle...

10 L'année suivante, le Groupe continue son examen de la situation en Uruguay et relève pour la première fois le cas d'une disparition d'enfant (E/CN.4/1492, § 145) :

[...] par une lettre datée du 17 novembre 1981, le Groupe de travail a communiqué au Gouvernement uruguayen un récit (émanant de l'un des grands parents de l'enfant en question selon lequel, d'après des témoins, le 18 mai 1978, une petite fille de 23 mois aurait été, ainsi que son père et sa mère, enlevée en un lieu non précisé de Montevideo, en Uruguay, par un groupe d'hommes armés, et selon lequel les parents et l'enfant seraient depuis manquants.

11 Le rapport, ainsi que les rapports suivants, font également état de la réponse des autorités de l'Uruguay, qui nient «toute complicité ou responsabilité » dans les disparitions, affirmant entreprendre les enquêtes nécessaires pour faire la lumière sur le sort des personnes disparues (E/CN.4/1983/14, § 93). 
12 Après le retour du Gouvernement civil, le Groupe de travail fait état d'informations de la société civile qui indiquent que si les prisonniers politiques ont été libérés, les disparus n'ont pas réapparu. Les ONG se plaignent par ailleurs de l'absence de tout progrès dans les enquêtes, tandis que le Gouvernement se prévaut de la création d'une Commission d'enquête sur les personnes disparues (E/CN.4/1986/18).

13 En 1987, le Groupe de travail a dans sa base de données 57 cas de disparitions forcées. Il fait état de l'adoption de la loi $\mathrm{n}^{\circ} 15.848$ sur la caducité de la prétention punitive de l'État (E/CN.4/1988/19, § 219, 222, 223 et suiv.).

14 En 1988, le Groupe de travail décide d'appliquer sa nouvelle règle dite de la " territorialité », en vertu de laquelle les cas des personnes enlevées sur le territoire d'un État sont recensés dans les statistiques consacrées à cet État, et non dans celui dont les forces sont présumées responsables, ou sur le territoire duquel la personne a été par la suite transférée. Il en résulte une diminution du nombre de cas au chapitre de l'Uruguay: de 57, on passe à 31 cas en suspens, les autres cas étant pour l'essentiel recensés dans les statistiques de l'Argentine (E/CN.4/1989/18, § 282 et 42). Sur un total de 39 cas intervenus sur le sol uruguayen ( 65 cas au total) à cette date, 8 cas ont été élucidés : 2 enfants retrouvés, 4 personnes en détention et 2 personnes en liberté.

Dans ses rapports suivants, le Groupe de travail indique avoir reçu quelques nouveaux cas et continue de suivre les développements en Uruguay sur la base des informations qui lui sont transmises : dossier de l'enquête dans l'affaire Elena Quinteros Almeida (affaire qui a fait par ailleurs l'objet de « constatations » du Comité des droits de l'homme en 1983 - il faut se rappeler que le $\mathrm{CDH}$ a joué un rôle très important à cette époque, puisque l'Uruguay avait fait « l'erreur » de ratifier le Protocole facultatif) (E/CN.4/1991/20, § 381), poursuite puis résolution de l'affaire de « l'enfant détenu en 1976 avec sa mère (alors qu'il était âgé de 20 jours) et qui avait par la suite disparu» (E/CN.4/1992/18, §352), dénonciation de la non-conformité au droit international de la loi de caducité, établissement de la Commission pour la paix et résultat des enquêtes menées, etc.

16 En 1995, 39 cas sont en suspens devant le Groupe. En 1996, le Groupe de travail mentionne à nouveau le retrait de 3 cas de disparitions des statistiques de l'Uruguay, dans la mesure où les personnes concernées avaient été enlevées sur le territoire de l'Argentine et où leur cas était déjà recensé dans les statistiques de l'Argentine (E/CN.4/1997/34, § 362). Même chose en 1997, avec 5 nouveaux cas retirés du dossier de l'Uruguay, au motif qu'il avait été établi que les disparitions avaient en fait eu lieu en Argentine (E/CN.4/1998/43, $\S 386)$. 31 cas restent donc en suspens devant le Groupe. Entre 1997 et aujourd'hui, 12 cas sont élucidés, dont un sur la base d'informations fournies par les sources et 11 sur la base d'informations fournies par le Gouvernement, ramenant le total des cas toujours sous examen à 19. Au total, depuis la création du Groupe de travail, ce sont donc 20 cas qui ont pu être élucidés, sur les 57 dont il était saisi au milieu des années 1980.

17 Le Groupe est toujours très prudent avec les chiffres et a coutume de mentionner le phénomène de sous-représentation des cas, en raison de plusieurs facteurs, notamment la peur des représailles ou tout simplement le fait que les victimes n'ont pas connaissance de la procédure. Dans le cadre de son mandat « humanitaire », le Groupe de travail n'a pas vocation à donner une vue complète de la situation des disparitions forcées dans un pays. Il donne ce type de vue d'ensemble dans le cadre des rapports qu'il établit à l'occasion d'une visite dans un pays. À ma connaissance, le Groupe de travail n'a pas demandé à 
effectuer une visite en Uruguay après 1980. Aujourd'hui, effectuer une telle visite aurait moins de sens, dans la mesure où la situation est désormais suivie de près par le Comité.

\section{Quelques points de droit important au regard de la situation en Uruguay}

18 À la lumière de la situation en Uruguay et dans d'autres pays, le Groupe de travail a rédigé plusieurs "observations générales", qui ont pour but d'interpréter de manière constructive la Déclaration de 1992 pour la protection de toutes les personnes contre les disparitions forcées. J'aimerais ici mentionner quatre de ces observations générales qui me paraissent particulièrement pertinentes dans le contexte actuel de l'Uruguay.

Dans son Observation générale sur l'article 18 de la Déclaration, le Groupe de travail a considéré

[qu'une] loi d'amnistie devrait être réputée contraire aux dispositions de la Déclaration, même si elle est entérinée par un référendum ou par une procédure de consultation similaire, dès lors que, directement ou indirectement, en conséquence de son application, elle produit l'un, quelconque ou la totalité des résultats suivants :

a) Éteindre les obligations qui incombent à l'État de mettre en examen, de poursuivre et de punir les personnes responsables des disparitions conformément aux articles 4, 13, 14 et 16 de la Déclaration; [...]

e) Abandonner les poursuites pénales ou clore les enquêtes contre les auteurs soupçonnés de faits de disparition ou imposer des peines négligeables pour donner à ces auteurs le bénéfice du droit de ne pas être jugés deux fois pour le même délit, ce qui reviendrait en fait à leur accorder l'immunité, en violation de l'article 4, paragraphe 1, de la Déclaration.

Par ailleurs, le Groupe de travail identifient également des «mesures similaires » à une amnistie, qui sont également contraires à la Déclaration, comme :

c) Appliquer des règles de prescription brèves ou dont le délai commence à courir alors même que le crime de disparition se poursuit, étant donné la nature continue de ce crime, en violation des articles 4 et 17 de la Déclaration ;

d) Appliquer des règles de prescription quand les faits de disparition constituent un crime contre l'humanité.

Dans son Observation générale sur la disparition forcée en tant que crime continu, le Groupe de travail a pris en compte l'évolution des pratiques nationales et internationales pour affirmer que

[...] le caractère continu de la disparition forcée a notamment pour conséquence qu'il est possible de condamner une personne pour un acte conduisant à une disparition forcée en invoquant un instrument juridique qui a été adopté après le début de la disparition forcée, nonobstant le principe fondamental de nonrétroactivité.

Autrement dit, il n'y a pas violation du principe de non-rétroactivité en matière pénale lorsqu'une personne est punie sur la base d'une loi adoptée postérieurement au commencement de la disparition forcée, pourvu que cette disparition forcée n'ait toujours pas été « élucidée » au moment de l'engagement des poursuites.

Enfin, dans son Observation générale sur le droit à la vérité dans le contexte des disparitions forcées, le Groupe de travail identifie un droit absolu des proches de connaître la vérité sur le sort des personnes disparues et le lieu où elles se trouvent. Ce droit ne peut faire l'objet d'aucune limitation ou dérogation. Le Groupe de travail 
considère que si la réalisation du droit à la vérité peut, dans des circonstances exceptionnelles, conduire à limiter le droit à la justice, cela ne peut être que dans les strictes limites énoncées dans son Observation générale sur l'article 18 de la Déclaration. Notamment, le Groupe de travail rappelle que

Le pardon ne devrait être accordé qu'après un processus de paix authentique ou des négociations de bonne foi avec les victimes, produisant pour résultat des excuses et l'expression de regrets de la part de l'État ou des auteurs des faits et des garanties pour prévenir les disparitions dans l'avenir.

Par ailleurs, le Groupe de travail a exprimé l'avis « qu'aucune limitation de ce type n'est admissible lorsque la disparition forcée constitue un crime contre l'humanité ».

S'agissant plus spécifiquement de l'Uruguay, je mentionnerai trois éléments complémentaires.

- Le 14 mai 2013, le Groupe de travail, conjointement avec trois autres mandats, a transmis une allégation générale concernant la clôture potentielle de certaines affaires judiciaires relatives à des cas de disparitions forcées, de torture et d'exécutions ayant eu lieu pendant la dictature, à la suite de plusieurs arrêts de la Cour suprême (Rapport de la $100^{\mathrm{e}}$ session A/ HRC/WGEID/100/1, §35). Les mêmes titulaires de mandats ont rendu leur démarche publique par un communiqué de presse du 25 juin 2013. Le 25 juillet 2013, le Gouvernement a répondu à cette communication de manière détaillée. Le Groupe de travail a formulé des observations dans son rapport de la $101^{\mathrm{e}}$ session (A/HRC/WGEID/101/1) et dans son dernier rapport annuel (A/HRC/27/49), rappelant notamment les dispositions de la Déclaration et des passages des observations générales que j'ai cités plus haut.

- Le rapport que l'Uruguay devait remettre en vertu de l'article 29 de la Convention a été examiné par le Comité contre les disparitions forcées, qui a rendu ses «observations finales » le 19 avril 2013. Dans ses observations, le Comité fait deux recommandations qui me paraissent particulièrement importantes à souligner. Premièrement, le Comité, tout en se déclarant satisfait que l'État ait introduit l'infraction de disparition forcée dans sa législation, s'est inquiété qu'il existait un large éventail entre les peines minimale et maximale prévues pour cette infraction. Il a recommandé de réduire cet éventail, de manière à ce que la peine minimale soit conforme à l'article 7 de la Convention et prenne donc en considération l'extrême gravité du crime. Deuxièmement, le Comité a demandé à l'État de

[...] veiller à ce que les faits de disparition forcée soient instruits comme tels et que les responsables soient punis pour cette infraction quel que soit le temps écoulé depuis le début de l'acte délictuel. [...] Le Comité tient à faire observer que l'infraction de disparition forcée revêt un caractère continu, conformément aux principes énoncés dans la Convention, et à rappeler les termes dans lesquels celle-ci définit le régime de prescription applicable aux actes de cette nature, ainsi qu'à souligner le caractère imprescriptible de cette infraction en cas de crime contre l'humanité. (Rapport annuel du Comité, A/68/56, p. 10-11)

- Enfin, le Rapporteur spécial sur la promotion de la vérité, de la justice, de la réparation et de la garantie de non répétition, Pablo de Greiff, a effectué une visite en Uruguay du 30 septembre au 4 octobre 2013. Dans son rapport de visite, le Rapporteur spécial considère que la «loi de caducité » est un obstacle dans le processus de justice transitionnelle en Uruguay :

El Relator Especial recalca que la Ley de Caducidad ha dado forma a la trayectoria de la justicia transicional en el Uruguay, representando un obstáculo, tanto real como simbólico, para lograr la rendición de cuentas por los crímenes del pasado. El Estado uruguayo no puede seguir avanzando ignorando esa realidad y los efectos que ha tenido en la vida de las 
víctimas, así como en cuanto al cumplimiento de sus obligaciones internacionales $y$ regionales en materia de derechos humanos. (A/HRC/27/56/Add.2, § 73)

Il recommande par ailleurs aux autorités d'éliminer tous les obstacles à la réalisation de la justice et d'assurer l'indépendance du pouvoir judiciaire et l'inamovibilité des juges :

a) Remover todos los obstáculos que impiden la presentación y el avance de las causas judiciales sin demoras indebidas, en cumplimiento del derecho a un recurso efectivo y otras normas internacionales de derechos humanos, incluyendo la sentencia de la Corte Interamericana de Derechos Humanos en el caso Gelman;

b) Avanzar en la reforma del poder judicial, asegurando que las disposiciones de la Ley Orgánica guarden armonía con los instrumentos internacionales de derechos humanos en materia de independencia judicial. En particular, los procedimientos que rigen los traslados, ascensos y sanciones de los jueces deben garantizar la independencia de los jueces y magistrados, para lo cual la transparencia, objetividad y la fundamentación de todas las decisiones son indispensables; tomar medidas en favor del establecimiento de un Consejo Superior de la Magistratura, encargado de la buena administración de las cortes, y de un Tribunal Superior Constitucional. (A/HRC/27/56/Add.2, § 75)

\section{AUTEUR}

\section{OLIVIER DE FROUVILLE}

Professeur à l'université Panthéon-Assas, directeur du Centre de recherche sur les droits de l'homme et le droit humanitaire, membre de l'Institut universitaire de France, membre du Comité des droits de l'homme des Nations unies et ancien président-rapporteur du Groupe de travail des Nations unies sur les disparitions forcées ou involontaires. 